\title{
Perspective
}

\section{The system capacity view of aging and longevity}

\author{
Jing-Dong J. Han*, Lei Hou ${ }^{\dagger}$, Na Sun ${ }^{\dagger}$, Chi Xu, Joseph McDermott and Dan Wang \\ Key Laboratory of Computational Biology, CAS Center for Excellence in Molecular Cell Science, Collaborative Innovation Center \\ for Genetics and Developmental Biology, Chinese Academy of Sciences-Max Planck Partner Institute for Computational Biology, \\ Shanghai Institutes for Biological Sciences, Chinese Academy of Sciences, Shanghai 200031, China \\ * Correspondence: jdhan@picb.ac.cn
}

Received March 15, 2017; Revised June 11, 2017; Accepted June 13, 2017

\begin{abstract}
Background: Aging is a complex systems level problem that needs a systems level solution. However, system models of aging and longevity, although urgently needed, are still lacking, largely due to the paucity of conceptual frameworks for modeling such a complex process.

Results: We propose that aging can be viewed as a decline in system capacity, defined as the maximum level of output that a system produces to fulfill demands. Classical aging hallmarks and anti-aging strategies can be well-aligned to system capacity. Genetic variants responsible for lifespan variation across individuals or species can also be explained by their roles in system capacity. We further propose promising directions to develop systems approaches to modulate system capacity and thus extend both healthspan and lifespan.

Conclusions: The system capacity model of aging provides an opportunity to examine aging at the systems level. This model predicts that the extent to which aging can be modulated is normally limited by the upper bound of the system capacity of a species. Within such a boundary, aging can be delayed by moderately increasing an individual's system capacity. Beyond such a boundary, increasing the upper bound is required, which is not unrealistic given the unlimited potential of regenerative medicine in the future, but it requires increasing the capacity of the whole system instead of only part of it.
\end{abstract}

Keywords: systems; system capacity; aging; longevity

\section{INTRODUCTION}

Aging is a major risk factor for most complex human diseases. It involves the decline of numerous biological functions or processes, such as stem cell renewal, nutrient sensing, autophagy, mitochondria, stress response and DNA damage response. Aging is therefore a systems level problem that needs a systems level strategy to tackle. However, systems level models of aging, although very much desired, are still lacking, largely due to the paucity of conceptual frameworks for modeling such a complex process.

Dietary restriction (DR) is a classical aging intervention strategy. DR has been found to extend lifespan in multiple species compared to ab libitum feeding (AL). However, its effect on lifespan can be affected substantially by genetic background [1,2], which suggests that even the assumed general anti-aging regimens need to be tailored for different individuals. Meanwhile, the paucity of longevity genes coming out of the major studies of centenarians [3-8] suggests that a non-genetic or epigenetic plasticity has a predominant effect on human longevity. These put forward new challenges to search for gene-gene interactions and gene-environment interactions through a systems biology approach in the study of human aging.

Calorie restriction (CR) and intermittent fasting (IF) are two major DR regimens in Caenorhabditis elegans. In our previous study that examined transcriptome changes caused by $\mathrm{CR} / \mathrm{IF}$ in $C$. elegans, we found three major patterns of changes that are modulated by $\mathrm{CR}$ and IF through aak-2/tax-6, rheb/tor-1 and daf-2/daf-16

These authors contributed equally to this work. 
pathways. They are related to i) (de)phosphorylation, ii) redox metabolism and unfolded protein response (UPR), and iii) cell cycle and DNA damage responses, respectively [9]. The maximal or minimal expression levels of gene expression groups altered by CR/IF suggest that they not only cause a delay in age-dependent changes, but also change the maximal or minimal levels of the gene functions, or capacities, of these gene functions (Figure 1). For instance, the post-reproduction stage seems to be a transition point for one of the three major transcriptome patterns during aging, PC2 (Figure 1B). The expression levels of cell cycle and DNA damage repair genes progressively increase during development, peak post

A
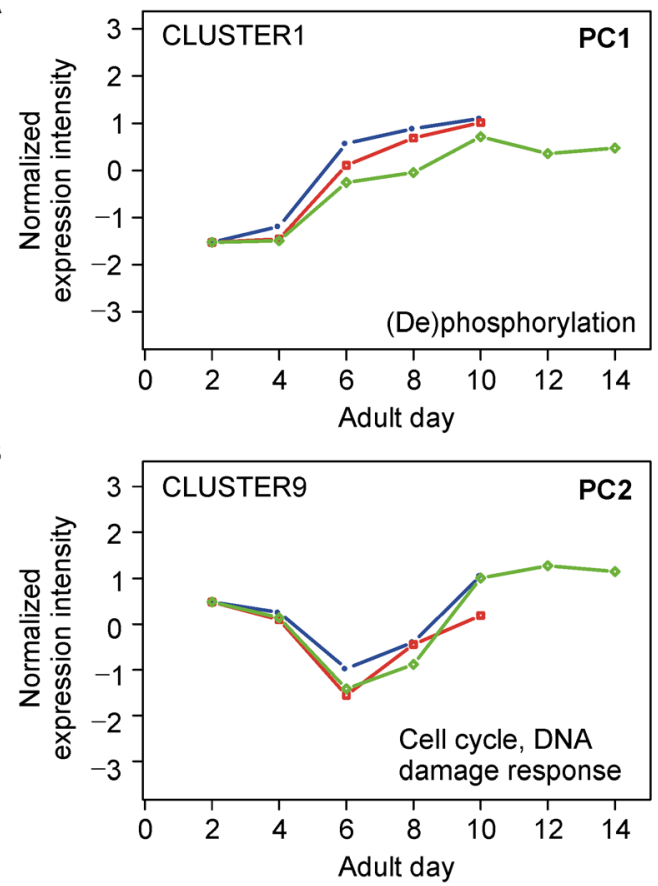

C

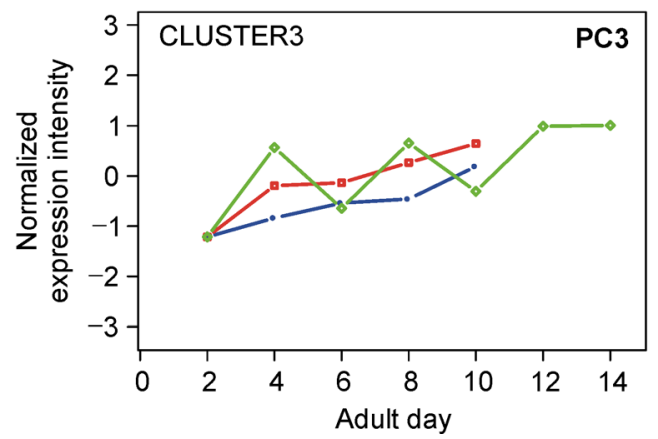

reproduction and decline afterwards [9]. DR/IF slightly and significantly shifted the maximal expression levels of these genes at the turning points (Figure 1C).

However, neither of the two DR regimens totally reverses or stops the reversed gene expression pattern or other major patterns during aging, but just delays the trend or slightly shifts the maximum (Figure 1). This indicates that the effect of $\mathrm{CR} / \mathrm{IF}$ and the maximal output of a system are limited by intrinsic factors. To further interpret such phenomena, we propose a concept of "system capacity" in aging, analogous to the "system capacity" concept that was put forward for manufacturing system automation [10]. We define system capacity as the
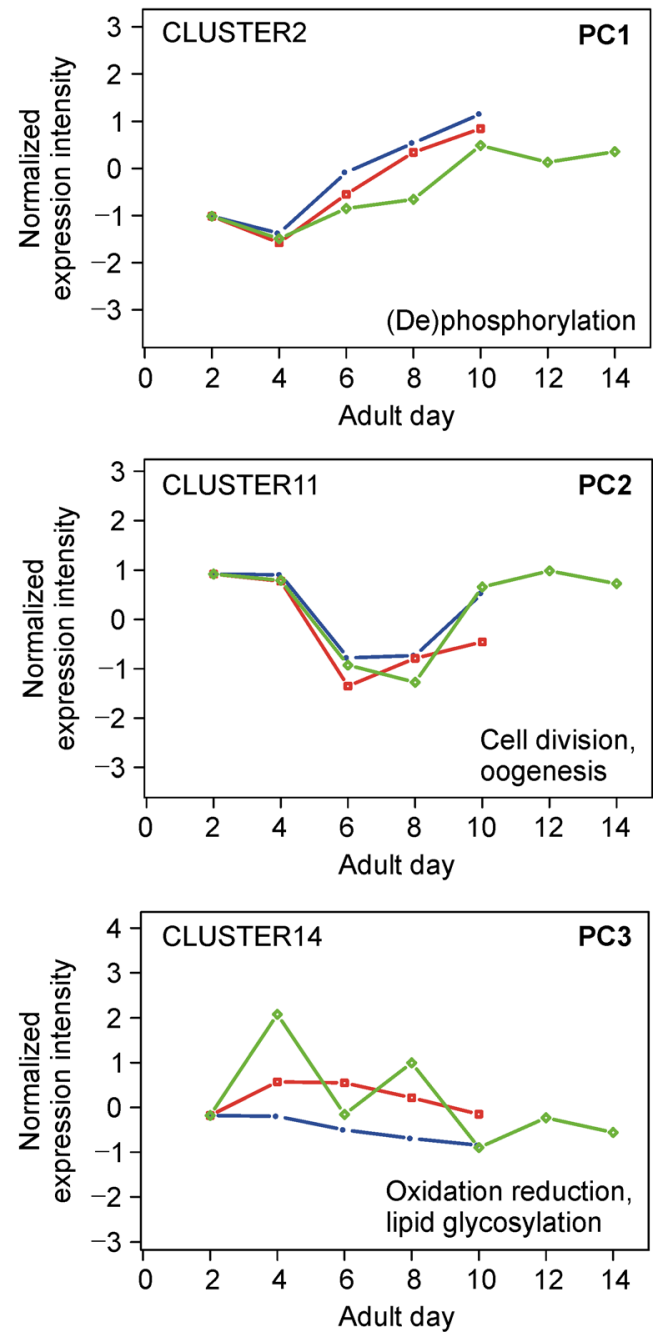

Figure 1. Gene expression profile changes during aging upon caloric restriction. Average gene expression profiles of clusters that change with age under ab libitum (AL) condition (blue curves) and two dietary restriction regimens, including calorie restriction (CR) (red curves) and intermittent fasting (IF) (green curves), adapted from Figure S1 in our previous study [9]. Gene ontology terms enriched in each gene cluster are shown. The CR/IF versus control transcriptome analysis over age revealed three major patterns of transcpritome changes that are modulated by CR and IF through the aak-2/tax-6, rheb/tor-1 and daf-2/daf-16 pathways, respectively: (A) (de)phosphorylation, (B) redox metabolism and unfolded protein response (UPR), and (C) cell cycle and DNA damage responses. The changed maximal or minimal expression levels of these gene-expression groups indicates that CR/IF not only causes a delay in age-dependent changes, but also a change in the maximal or minimal levels of gene functions. 
maximum level of output that a system produces to fulfill demands. The system capacity of a whole organism is maintained through the capacity of many components (or subsystems) and the interactions among them, and both components and their interactions are subject to the capacity limits of regulatory system (Figure 2A). Systems capacity can be divided into two distinct types of functions: i) production of energy and material and ii) homeostasis or maintenance of the system that consists of information flow and control (Figure 2A). As an example, the maximal velocity, but not the average velocity, is a reflection of the system capacity in motility [11].

Although there are debates on what measures aging, the commonly agreed upon standard is lifespan. An alternative could be healthspan, but this is harder to measure. So here the output of an organismal-level system includes all the products, energy, and information a system needs to generate to maintain life. Meanwhile, for different components of a system, or subsystems, their outputs can be different. For example, the output of the mitochondria system is mainly energy, whereas the output of the DNA repair system is repaired DNA.

Similarly, demands here refer to all the demands a system needs to handle in order to maintain life. For different components, the demands can also be different, for example, for the DNA repair system, the demands are the total number of damaged DNA molecules to be repaired, and for the immune system, the demands are the total external pathogens, and damaged or dead cells, that need to be cleared.

Of course, not all components and their capacity should be equally weighted. Instead, they should be weighted in terms of their contribution to the lifespan of an organism. As an example for simplicity, by a linear formula of lifespan, $L=\Sigma \alpha_{i} C_{i}$, where $C_{i}$ is the $i$ th component that significantly contributes to the lifespan of an organism, and $\alpha_{i}$ is the weight of contribution of by $C_{i}$.

With the system capacity model, we can i) explain the well-established aging hallmarks [12], ii) gauge the ways, and to what extent, system capacity and hence the aging process can be modulated, iii) predict new components that may affect aging and longevity, iv) predict aging regulators from their importance to the genetic networks, and their overall effect on stress resistance, and v) eventually when the quantification of major components and interactions is available, to theoretically predict lifespan and death (breakdown of a system) of a specific system.

\section{AGING IN A VIEW OF SYSTEM CAPACITY}

To examine aging at the systems level, we draw an analogy of an organism to a manufacturing system. For a manufacturing system to operate, system dynamics are largely determined by demand from markets, machine capacity and decision to invest or not to invest in capacity. Similarly, in a biological system, system dynamics are mainly determined by the balance of physiological demand versus the system capacity to fulfill this demand, and ability of the decision and regulatory circuitry, such as sensing and signaling pathways to tune system capacity and the balance between the demands and system capacity based on the demand perceived (Figure 2B). Physiological demand comes from two sources: extrinsic factors, such as nutrients and temperature; intrinsic factors, such as growth and repair. However, not all physiological demand would be met due to limited resources or regulation ability, so the unfulfilled demand in turn drives decision machinery to judge whether system capacity should be extended. Nevertheless, the increase of system capacity may not solve the problem, especially when the system already maxed out its capacity, and, thus, overload factors begin to take control. Failure rate will then be non-negligible and also spread to undermine the system capacity and confound the signaling in and out from the decision and regulatory circuitry, which activates potential positive feedback, making it more fragile and inevitably moving towards system capacity decline, which is "aging" of a biological system. As a result of aging, increasing unfulfilled demand will accumulate and most of which, if not all, finally pushes organisms to death or to the breakdown of a biological system.

Evolutionary selection has ensured the demand is largely met by system capacity until passing reproduction age; afterwards, although the unfulfilled demand starts to increase - as shown by the trend of PC1 which largely reflects the expression changes of metabolic genes (Figure 1B) - the system is not under evolutionary selection through reproduction to increase system capacity (Figure 1A). Although the decline of system capacity and increase of unfulfilled demand seems to be inevitable, system capacity is tunable through the selected pre-reproduction regulatory systems, which largely determines the tunability of the aging process.

\section{AGING HALLMARKS AS SYSTEM CAPACITY DECLINES}

Nine potential hallmarks of aging have been summarized [12], that are grouped into three classes: "primary hallmarks" including "genomic instability", "telomere attrition", "loss of proteostasis", and "epigenetic alterations"; "antagonistic hallmarks" including "deregulated nutrient sensing", "mitochondrial dysfunction", and "cellular senescence"; "integrative hallmarks" including "stem cell exhaustion", and "altered intercellular 
A

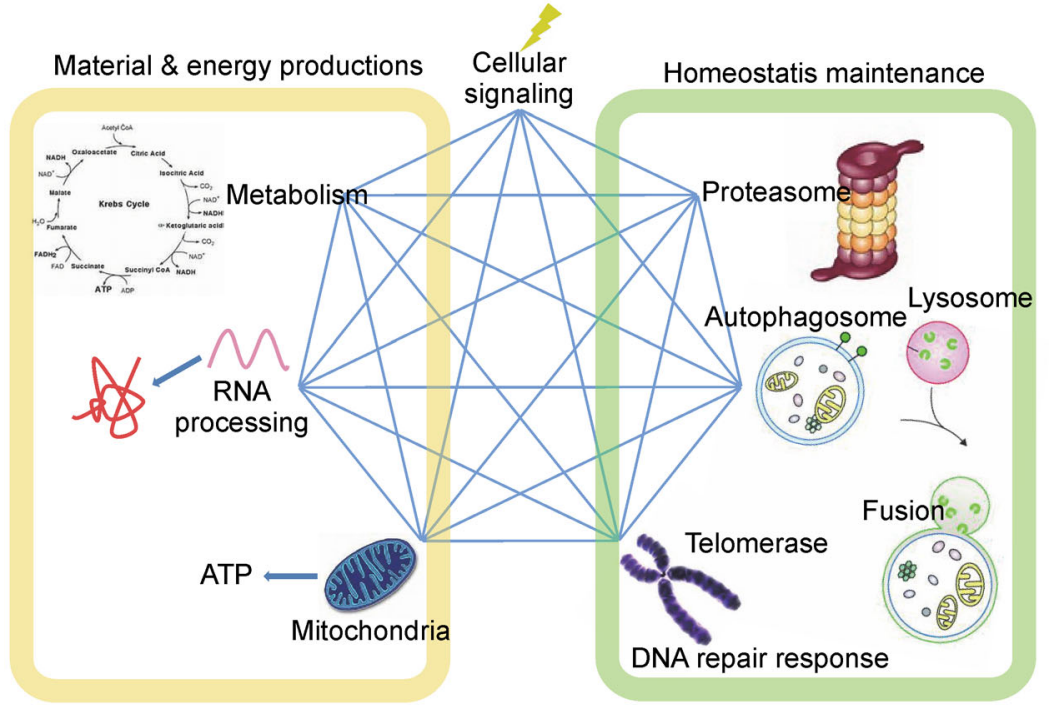

B

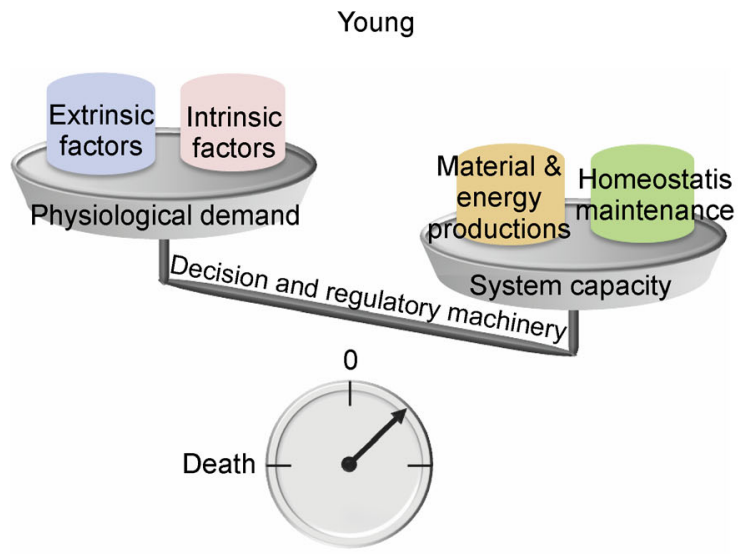

Fulfilled demand

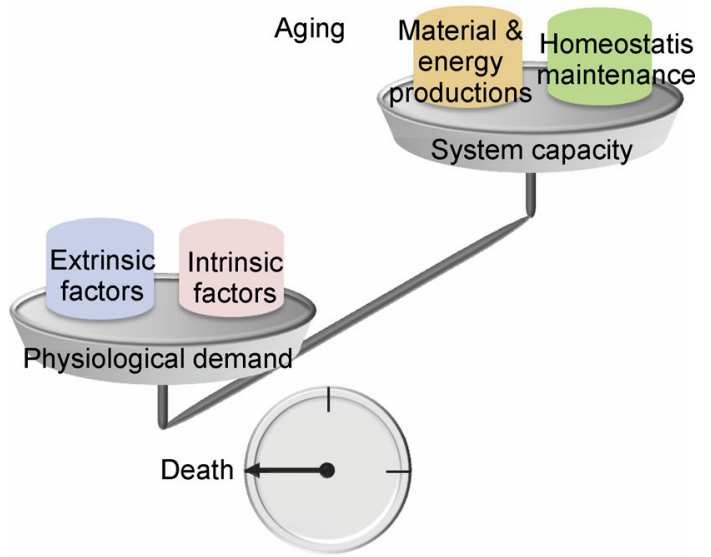

Unfulfilled demand

C

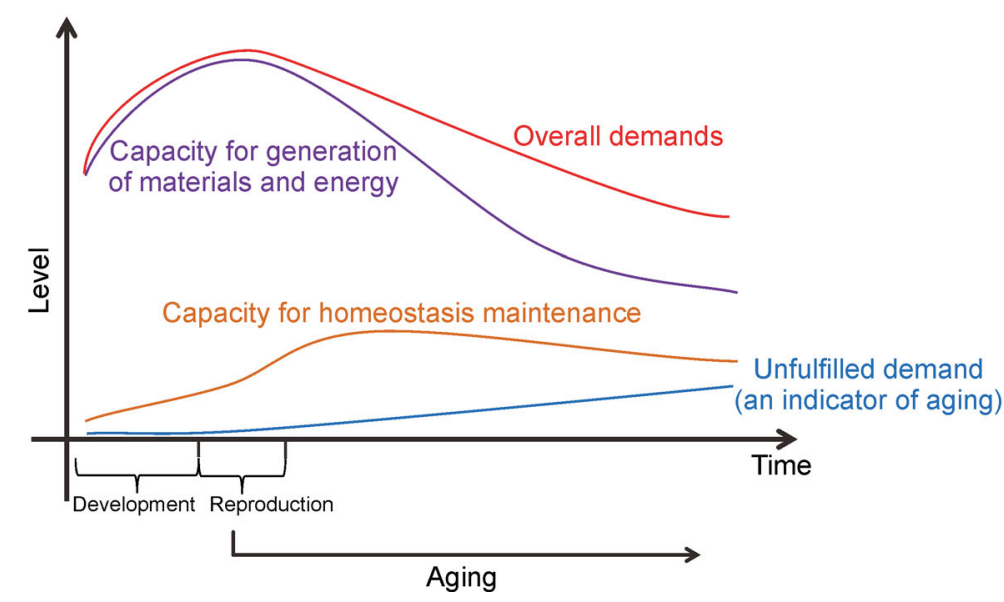

Figure 2. System capacity and its dynamics in aging. (A) System capacity consists of two types of components: materials and energy production, and homeostatic maintenance, together with the interactions and regulations among these components. (B) Balance of system capacity versus demands. When an animal is young, decision and regulatory circuitry and machineries constituting system capacity are both efficient enough to meet most if not all physiological demand; when it ages, decreased system capacity or less responsive regulatory circuitry lead to overload, resulting in increasing failure rate, which in turn undermines decision and regulatory circuitry and system capacity and further increases unfulfilled demand, which ultimately leads to death. (C) Dynamics of system capacity throughout life. 
communication".

These hallmarks are actually well-aligned to our system capacity concept (Table 1). According to the definition of system capacity, "genomic instability", "telomere attrition" and "loss of proteostasis" are related to selfmaintenance programs and "mitochondrial dysfunction "is related to energy production, so all can be characterized as dysfunction of components that maintain system capacity. Meanwhile, "deregulated nutrient sensing", "altered intercellular communication", and "epigenetic alterations" are related to signaling and regulation, and thus are parts of decision and regulatory circuitry. The rest of the hallmarks including "cellular senescence" and "stem cell exhaustion", are cellular consequences of unfulfilled demand or capacity decline. Together, these hallmarks can be classified according to whether they are components for maintaining system capacity, factors to regulate it or balance it with demands, or the consequence of exceeding it. From such a perspective, modulating system capacity modulates aging.

Although system capacity is under developmentally programmed regulation before reproduction, it is tunable before or after reproduction. Because the regulation is selected for development, not for aging, timing is important: an intervention might be only effective at a specific time period in lifespan. For example, inactivating mitochondrial function only during early development, but not later, dose-dependently increases lifespan [13]. As another example, $h s f-1$ is required during early development, whereas daf-16 is required afterwards, to counter proteotoxicity [14]. In addition, different components of each part are interconnected: modulation of one hallmark could actually lead to a chain of alterations of other hallmarks. For example, nutrient sensing will affect protein homeostasis [15], epigenetic regulation could modulate mitochondria functions [16], mitochondria dysfunction will increase ROS, which in turn affects homeostasis [17-19]. Taken together, timing or interconnectivity can affect whether a component of the system capacity is able to modulate aging or not.

\section{ANTI-AGING STRATEGIES AS A MEANS TO INCREASE SYSTEM CAPACITY OR BALANCE IT VERSUS DEMAND}

The strategies that postpone aging are more desirable than the opposite, and are more difficult to develop, which is consistent with the destruction of a system being far easier to achieve than increasing system capacity.

Most anti-aging strategies can be aligned to targeting components that constituting the system capacity. Some directly target the capacity machinery, such as mitochondria [20] and proteostasis components [21], or telomerase [22]. Some work through decision and regulatory circuitry, such as through epigenetic regulators such as sirtuins and histone modification enzymes [23-26], and signaling pathways [15]. Whereas healthy lifestyle strategies can directly reduce the physiological demands to remove damaging materials, such as reduction of environmental exposure to UV light or pollution, etc.

Some lifestyle interventions may also modulate aging by targeting multiple components and signaling pathways. Our laboratory showed that based on a crosssectional microarray design, exercise together with dietary choices including high/low fat diet and AL/DR, may affect mouse lifespan through multiple components, including the oxidative phosphorylation, ribosome, peroxisome, lysosome, apoptosis and inflammation pathways [27]. A detailed time course study of $C$. elegans aging upon AL and DR regimens indicates that RHEB-1, AAK-2, and DAF-16 related pathways play roles in lifespan regulation by CR/IF. The study also demonstrated that simultaneous modulation of all three pathways can not only recapitulate DR's effect, but can be even more effective than DR itself in lifespan extension. The success of reverse engineering DR is only a starting point. It sheds lights on a promising direction for future antiaging strategies: systematic perturbations on multiple parts will target aging more effectively and have fewer side effects than single point perturbations.

Table 1. Mapping of aging hallmarks to the system capacity model of aging.

\begin{tabular}{|c|c|c|}
\hline \multicolumn{2}{|c|}{ Components in the model of Figure $2 \mathrm{~B}$} & \multirow{2}{*}{$\frac{\text { Aging hallmarks }}{\text { Genomic instability }}$} \\
\hline System capacity & Homeostatic maintenance & \\
\hline & & Telomere attrition \\
\hline & & Loss of proteostasis \\
\hline & Materials \& energy production & Mitochondria dysfunction \\
\hline \multirow{3}{*}{\multicolumn{2}{|c|}{ Decision and regulatory machinery }} & Deregulated nutrient sensing \\
\hline & & Altered intercellular communication \\
\hline & & Epigenetic alterations \\
\hline \multirow{2}{*}{\multicolumn{2}{|c|}{ Unfulfilled demand }} & Cellular senescence \\
\hline & & Stem cell exhaustion \\
\hline
\end{tabular}


The effects of these strategies mainly fall into three categories (Figure 3): i) slowing down system capacity decline or reducing the rate the aging-related functional decay relative to demands (Figure $3 \mathrm{~A}$ ); ii) presetting the initial parameters of system capacities of components (or subsystems) to a higher level at the beginning of life or before the start of aging, relative to demands; and iii) resetting the overall system capacity back to the young
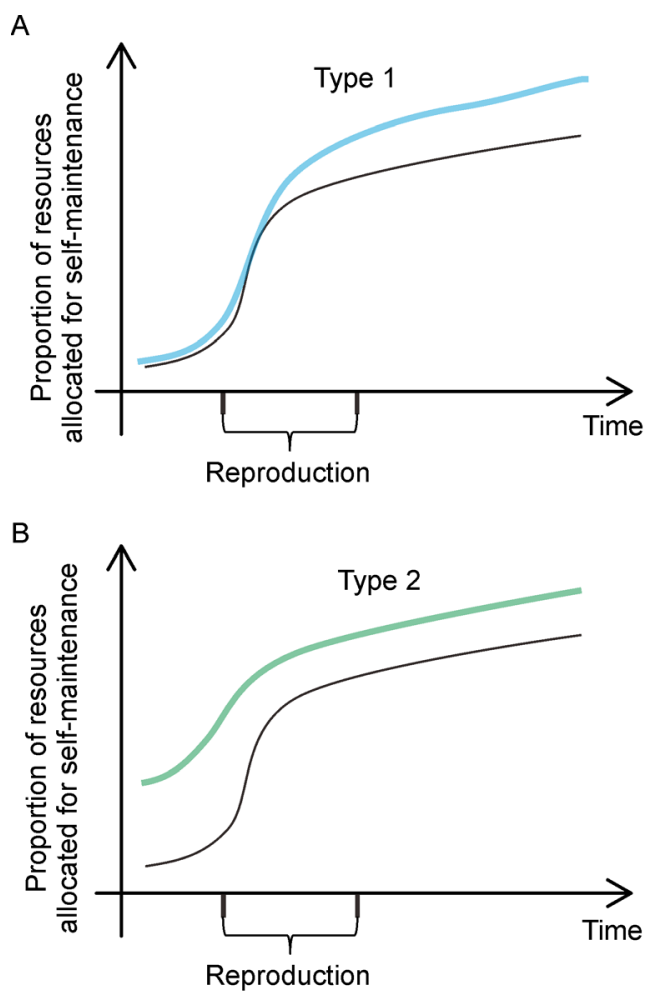

C

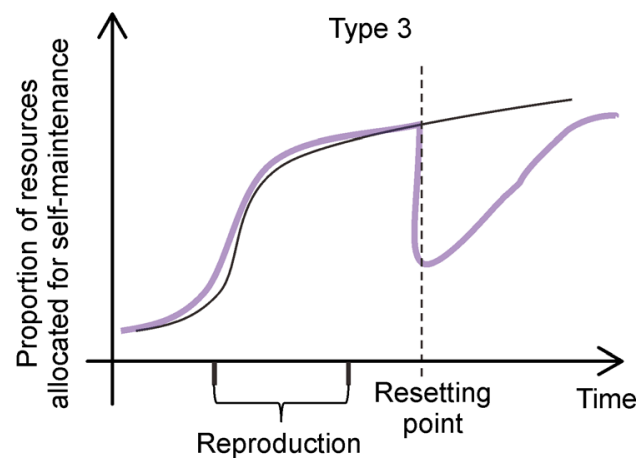

Figure 3. Different types of anti-aging strategies from the perspective of system capacity. (A) Type 1: More resources are allocated for system capacity selfmaintenance from reproduction and onward to reduce the rate of aging-related functional decay relative to demands. (B) Type 2: The initial parameters of system capacity are preset to a higher level relative to demands. (C) Type 3: The overall system capacity is reset to the young state. state (Figure 3C). Both i) and ii) can be achieved through targeting the different system components of an organism (such as energy and material generation and selfmaintenance components), or through reducing the intrinsic (such as reproduction and metabolism) and extrinsic demands (such as exposure to damaging materials) (Figure 3B). Most strategies fall into the first category, including genetic, pharmaceutical and dietary perturbations through nutrient-sensing pathways, stress response pathways, sirtuins or some epigenetic factors to boost self-maintenance programs [9,15,28-31]. Mitochondria defects during development in C. elegans, which probably shape the system capacity through mitohormesis, probably belong to the second category [20]. Parabiosis and reprogramming to induced pluripotent stem cell (iPSC) are two approaches to rejuvenation at the cellular level, and thus could be classified into the third category, although the mechanisms are still dubious [3234]. Obviously, the first and third categories are more practical to achieve without any side-effects on proper development.

\section{LIFESPAN VARIATIONS AS A REFLECTION OF SYSTEM CAPACITY DIFFERENCES}

Besides the external anti-aging perturbations, comparative studies between short-lived and long-lived individuals within one species or across different species provide additional insights into the relationship between system capacity and aging.

Regarding individual variations, numerous genomewide association studies (GWAS) have been carried out to explore the genetic loci that are associated with longevity in human, especially centenarians. Only a few genes are identified as robust results: FOXO1A and FOXO3A responsible for stress response and DNA damage [5,6]; $A P O E$ in cholesterol homeostasis [3,4,7]; MINPP1, involved in inositol phosphate metabolism [8]. Interestingly, some of them are likely to function as components of self-maintenance machinery or regulatory circuitry, and their reinforcement could lead to a higher system capacity and thus longer maximal lifespan of a species. The recent finding of a limited maximal human lifespan based on evidence of a plateau in maximum age in recent decades suggests that the variations in maximal lifespan within a species are relatively very small [35], in contrast to the variations in lifespan across species.

The results of comparisons across different species are more complex. For example, many studies examine different rodents, among which the lifespan can differ by ten-fold: two or three years for mouse or rat, and twenty or thirty years for blind mole-rat or naked mole-rat. Multiple alterations of genes or pathways contribute to their 
lifespan differences, including p16-INK4A or p53 in tumor suppression machinery [36-38], oxidative stress resistance [39,40], translation fidelity [41], autophagy $[42,43]$, and insulin and mTOR signaling pathways [44,45], Ifnbl and $M x I$ in the interferon signaling pathway [46], and so on. In addition, other studies on longevity in large animals or flying long-lived animals also reveal a mixed pattern of strategies - interventions of both components in system capacity machinery and decision and regulatory circuitry. It indicates that multiple components might have been modulated at the systems level to optimize fitness during evolution, and thus much larger lifespan variations across different species.

\section{TESTABLE HYPOTHESES BASED ON THE SYSTEM CAPACITY MODEL}

The systems capacity model of aging leads to testable hypotheses. Those that have been already established as true lend support to our model and those not yet tested will guide future research.

i) Any component of system capacity that is essential to maintain life and has its own capacity limit reached will have a consequence on the aging process, and can be a trigger for aging even if other parts are not yet reaching their limits. For example, RNA processing has not been proposed as a hallmark of aging, but its capacity is indeed limited during the aging process, and it is essential for maintaining life. Therefore, we would predict from our model, that when RNA processing reaches its capacity limit, it will trigger the system towards decline or aging. Indeed, splicing has just been shown to an important process regulating aging and lifespan [47].

ii) Regulatory/signaling networks connecting components of system capacity also have limited capacities. When their capacities are reached, the regulatory networks lose their regulation precision. Increased gene expression noise has indeed been observed during aging, which is an indication of overload on the regulatory network capacity [48]. A prediction from our model would be that the loss of regulatory precision would also lead to aging, that is, introducing random noises to the regulation output will lead the system towards decline or aging, a hypothesis that can be tested both computationally and experimentally.

iii) As a system is composed of many components whose capacities are limited, the more components a gene interacts with or the more components' capacities a gene regulates, the more likely it will affect the whole system and hence its overall capacity. This is consistent with the fact that aging and lifespan regulators are frequently hubs in the molecular interaction networks [49-51], and the fact that pleiotropic genes and genes regulating general stress tolerance in many physiological components or processes, such as daf-16, are key regulators of aging and longevity.

iv) Based on the model, if the relative level of demand (both extrinsic and intrinsic) and the system capacity (in material/energy production and homeostasis maintenance) are known for an individual organism, the probability of death for this given individual can be quantitatively predicted. Although this requires a myriad of data collection, our model points to the direction of what data needs to be collected (that is, quantification of systems capacity components and demands) and the feasibility of finally being able to predict death.

v) An extension for the fourth prediction will be development of anti-aging strategies targeting different parts of system capacity, or aspects of aging, when there is a precise diagnosis of which component of the system is running over capacity. Its efficacy will be dependent on whether it can balance the specific loss of the capacity for a particular component. Therefore a precision medicine approach is needed to first diagnose where system capacity is lost and then match an intervention that specifically addresses the lost capacity, or reduces the demand that burdens the specific capacity, and to regain the balance between demand and capacity, hence achieving the goal of healthy aging.

\section{CONCLUSIONS AND OUTLOOK}

The system capacity model of aging provides an opportunity to examine aging at the systems level. This model predicts that the extent to which aging can be modulated is normally limited by the upper bound of the system capacity of a species. Within such a boundary, aging can be delayed by moderately increasing an individual's system capacity. Beyond such a boundary, increasing the upper bound is required, which is not unrealistic given the unlimited potential of regenerative medicine in the future, but it requires increasing the capacity of the whole system instead of only part of it.

The system capacity model provides a framework for applying systems approaches in at least four directions: i) identifying components whose capacity limits are maximized or overload during aging, ii) modeling regulatory circuits to examine their effects on the aging process, iii) quantifying the level of components versus demands and building a computational model to predict lifespan and death of a system, and iv) diagnosing capacity deficiency of a system and rebalancing demands at the individual level through combinatorial interventions for healthy lifespan.

\section{ACKNOWLEDGEMENTS}

We thank Yizhen Yan for illustration suggestions and acknowledge supports 
from China Ministry of Science and Technology 2015CB964803 and 2016YFE0108700 and the National Natural Science Foundation of China 91329302, 31210103916 and 91519330, and Chinese Academy of Sciences XDB19020301 and XDA01010303 to J.D.J.H.

\section{COMPLIANCE WITH ETHICS GUIDELINES}

The authors Jing-Dong J. Han, Lei Hou, Na Sun, Chi Xu, Joseph McDermott and Dan Wang declare that they have no conflict of interests.

This article is a perspective article and does not contain any studies with human or animal subjects performed by any of the authors.

\section{REFERENCES}

1. Liao, C. Y., Rikke, B. A., Johnson, T. E., Diaz, V. and Nelson, J. F. (2010) Genetic variation in the murine lifespan response to dietary restriction: from life extension to life shortening. Aging Cell, 9, 92-95

2. Schleit, J., Johnson, S. C., Bennett, C. F., Simko, M., Trongtham, N., Castanza, A., Hsieh, E. J., Moller, R. M., Wasko, B. M., Delaney, J. R., et al. (2013) Molecular mechanisms underlying genotype-dependent responses to dietary restriction. Aging Cell, $12,1050-1061$

3. Beekman, M., Blanché, H., Perola, M., Hervonen, A., Bezrukov, V., Sikora, E., Flachsbart, F., Christiansen, L., De Craen, A. J., Kirkwood, T. B., et al. (2013) Genome-wide linkage analysis for human longevity: genetics of healthy aging study. Aging Cell, 12, 184-193

4. Deelen, J., Beekman, M., Uh, H. W., Helmer, Q., Kuningas, M., Christiansen, L., Kremer, D., van der Breggen, R., Suchiman, H. E., Lakenberg, N., et al. (2011) Genome-wide association study identifies a single major locus contributing to survival into old age; the APOE locus revisited. Aging Cell, 10, 686-698

5. Flachsbart, F., Caliebe, A., Kleindorp, R., Blanché, H., von EllerEberstein, H., Nikolaus, S., Schreiber, S. and Nebel, A. (2009) Association of $\mathrm{FOXO3A}$ variation with human longevity confirmed in German centenarians. Proc. Natl. Acad. Sci. USA, 106, 2700-2705

6. Li, Y., Wang, W. J., Cao, H., Lu, J., Wu, C., Hu, F. Y., Guo, J., Zhao, L., Yang, F., Zhang, Y. X., et al. (2009) Genetic association of FOXO1A and FOXO3A with longevity trait in Han Chinese populations. Hum. Mol. Genet., 18, 4897-4904

7. Nebel, A., Kleindorp, R., Caliebe, A., Nothnagel, M., Blanché, H., Junge, O., Wittig, M., Ellinghaus, D., Flachsbart, F., Wichmann, H. E., et al. (2011) A genome-wide association study confirms $A P O E$ as the major gene influencing survival in long-lived individuals. Mech. Ageing Dev., 132, 324-330

8. Newman, A. B., Walter, S., Lunetta, K. L., Garcia, M. E., Slagboom, P. E., Christensen, K., Arnold, A. M., Aspelund, T., Aulchenko, Y. S., Benjamin, E. J., et al. (2010) A meta-analysis of four genome-wide association studies of survival to age 90 years or older: the cohorts for heart and aging research in genomic epidemiology consortium. J. Gerontol. A Biol. Sci. Med. Sci., 65A, 478-487

9. Hou, L., Wang, D., Chen, D., Liu, Y., Zhang, Y., Cheng, H., Xu, C., Sun, N., McDermott, J., Mair, W. B., et al. (2016) A systems approach to reverse engineer lifespan extension by dietary restriction. Cell Metab., 23, 529-540

10. Špicar, R. (2014) System dynamics archetypes in capacity planning. Procedia Eng., 69, 1350-1355

11. Hahm, J. H., Kim, S., DiLoreto, R., Shi, C., Lee, S. J., Murphy, C. T. and Nam, H. G. (2015) C. elegans maximum velocity correlates with healthspan and is maintained in worms with an insulin receptor mutation. Nat. Commun., 6, 8919

12. López-Otín, C., Blasco, M. A., Partridge, L., Serrano, M. and Kroemer, G. (2013) The hallmarks of aging. Cell, 153, 1194-1217

13. Rea, S. L., Ventura, N. and Johnson, T. E. (2007) Relationship between mitochondrial electron transport chain dysfunction, development, and life extension in Caenorhabditis elegans. PLoS Biol., 5, e259

14. Cohen, E., Du, D., Joyce, D., Kapernick, E. A., Volovik, Y., Kelly, J. W. and Dillin, A. (2010) Temporal requirements of insulin/IGF-1 signaling for proteotoxicity protection. Aging Cell, 9, 126-134

15. Kenyon, C. J. (2010) The genetics of ageing. Nature, 464, 504-512

16. Hashizume, O., Ohnishi, S., Mito, T., Shimizu, A., Ishikawa, K., Nakada, K., Soda, M., Mano, H., Togayachi, S., Miyoshi, H., et al. (2015) Epigenetic regulation of the nuclear-coded GCAT and SHMT2 genes confers human age-associated mitochondrial respiration defects. Sci. Rep., 5, 10434

17. Edgar, D., Shabalina, I., Camara, Y., Wredenberg, A., Calvaruso, M. A., Nijtmans, L., Nedergaard, J., Cannon, B., Larsson, N. G. and Trifunovic, A. (2009) Random point mutations with major effects on protein-coding genes are the driving force behind premature aging in mtDNA mutator mice. Cell Metab., 10, 131138

18. Hiona, A., Sanz, A., Kujoth, G. C., Pamplona, R., Seo, A. Y., Hofer, T., Someya, S., Miyakawa, T., Nakayama, C., SamhanArias, A. K., et al. (2010) Mitochondrial DNA mutations induce mitochondrial dysfunction, apoptosis and sarcopenia in skeletal muscle of mitochondrial DNA mutator mice. PLoS One, 5, e11468

19. Sena, L. A. and Chandel, N. S. (2012) Physiological roles of mitochondrial reactive oxygen species. Mol. Cell, 48, 158-167

20. Dillin, A., Hsu, A. L., Arantes-Oliveira, N., Lehrer-Graiwer, J., Hsin, H., Fraser, A. G., Kamath, R. S., Ahringer, J. and Kenyon, C. (2002) Rates of behavior and aging specified by mitochondrial function during development. Science, 298, 2398-2401

21. Vilchez, D., Morantte, I., Liu, Z., Douglas, P. M., Merkwirth, C., Rodrigues, A. P., Manning, G. and Dillin, A. (2012) RPN-6 determines $C$. elegans longevity under proteotoxic stress conditions. Nature, 489, 263-268

22. Bernardes de Jesus, B., Vera, E., Schneeberger, K., Tejera, A. M., Ayuso, E., Bosch, F. and Blasco, M. A. (2012) Telomerase gene therapy in adult and old mice delays aging and increases longevity without increasing cancer. EMBO Mol. Med., 4, 691-704

23. Greer, E. L., Maures, T. J., Hauswirth, A. G., Green, E. M., Leeman, D. S., Maro, G. S., Han, S., Banko, M. R., Gozani, O. and Brunet, A. (2010) Members of the H3K4 trimethylation complex regulate lifespan in a germline-dependent manner in C. elegans. Nature, 466, 383-387

24. Jin, C., Li, J., Green, C. D., Yu, X., Tang, X., Han, D., Xian, B., Wang, D., Huang, X., Cao, X., et al. (2011) Histone demethylase 
UTX-1 regulates C. elegans life span by targeting the insulin/IGF1 signaling pathway. Cell Metab., 14, 161-172

25. Satoh, A., Brace, C. S., Rensing, N., Cliften, P., Wozniak, D. F., Herzog, E. D., Yamada, K. A. and Imai, S. (2013) Sirt1 extends life span and delays aging in mice through the regulation of Nk2 homeobox 1 in the DMH and LH. Cell Metab., 18, 416-430

26. Viswanathan, M. and Guarente, L. (2011) Regulation of Caenorhabditis elegans lifespan by sir-2.1 transgenes. Nature, 477, E1-E2

27. Zhou, B., Yang, L., Li, S., Huang, J., Chen, H., Hou, L., Wang, J., Green, C. D., Yan, Z., Huang, X., et al. (2012) Midlife gene expressions identify modulators of aging through dietary interventions. Proc. Natl. Acad. Sci. USA, 109, E1201-E1209

28. Burkewitz, K., Zhang, Y. and Mair, W. B. (2014) AMPK at the nexus of energetics and aging. Cell Metab., 20, 10-25

29. Greer, E. L. and Brunet, A. (2009) Different dietary restriction regimens extend lifespan by both independent and overlapping genetic pathways in C. elegans. Aging Cell, 8, 113-127

30. Johnson, S. C., Rabinovitch, P. S. and Kaeberlein, M. (2013) mTOR is a key modulator of ageing and age-related disease. Nature, 493, 338-345

31. Longo, V. D. and Kennedy, B. K. (2006) Sirtuins in aging and agerelated disease. Cell, 126, 257-268

32. Loffredo, F. S., Steinhauser, M. L., Jay, S. M., Gannon, J., Pancoast, J. R., Yalamanchi, P., Sinha, M., Dall'Osso, C., Khong, D., Shadrach, J. L., et al. (2013) Growth differentiation factor 11 is a circulating factor that reverses age-related cardiac hypertrophy. Cell, 153, 828-839

33. Miller, J. D., Ganat, Y. M., Kishinevsky, S., Bowman, R. L., Liu, B., Tu, E. Y., Mandal, P. K., Vera, E., Shim, J. W., Kriks, S., et al. (2013) Human iPSC-based modeling of late-onset disease via progerin-induced aging. Cell Stem Cell, 13, 691-705

34. Studer, L., Vera, E. and Cornacchia, D. (2015) Programming and reprogramming cellular age in the era of induced pluripotency. Cell Stem Cell, 16, 591-600

35. Dong, X., Milholland, B. and Vijg, J. (2016) Evidence for a limit to human lifespan. Nature, 538, 257-259

36. Ashur-Fabian, O., Avivi, A., Trakhtenbrot, L., Adamsky, K., Cohen, M., Kajakaro, G., Joel, A., Amariglio, N., Nevo, E. and Rechavi, G. (2004) Evolution of p53 in hypoxia-stressed Spalax mimics human tumor mutation. Proc. Natl. Acad. Sci. USA, 101, 12236-12241

37. Avivi, A., Ashur-Fabian, O., Joel, A., Trakhtenbrot, L., Adamsky, K., Goldstein, I., Amariglio, N., Rechavi, G. and Nevo, E. (2007) P53 in blind subterranean mole rats - loss-of-function versus gain-of-function activities on newly cloned Spalax target genes. Oncogene, 26, 2507-2512

38. Seluanov, A., Hine, C., Azpurua, J., Feigenson, M., Bozzella, M., Mao, Z., Catania, K. C. and Gorbunova, V. (2009) Hypersensitivity to contact inhibition provides a clue to cancer resistance of naked mole-rat. Proc. Natl. Acad. Sci. USA, 106, 19352-19357

39. Andziak, B., O'Connor, T. P., Qi, W., DeWaal, E. M., Pierce, A.,
Chaudhuri, A. R., Van Remmen, H. and Buffenstein, R. (2006) High oxidative damage levels in the longest-living rodent, the naked mole-rat. Aging Cell, 5, 463-471

40. Pérez, V. I., Buffenstein, R., Masamsetti, V., Leonard, S., Salmon, A. B., Mele, J., Andziak, B., Yang, T., Edrey, Y., Friguet, B., et al. (2009) Protein stability and resistance to oxidative stress are determinants of longevity in the longest-living rodent, the naked mole-rat. Proc. Natl. Acad. Sci. USA, 106, 3059-3064

41. Azpurua, J., Ke, Z., Chen, I. X., Zhang, Q., Ermolenko, D. N., Zhang, Z. D., Gorbunova, V. and Seluanov, A. (2013) Naked molerat has increased translational fidelity compared with the mouse, as well as a unique $28 \mathrm{~S}$ ribosomal RNA cleavage. Proc. Natl. Acad. Sci. USA, 110, 17350-17355

42. A. Rodriguez, K., Wywial, E., I. Perez, V., J. Lambert, A., H. Edrey, Y., N. Lewis, K., Grimes, K., L. Lindsey, M., D. Brand, M. and Buffenstein, R. (2011) Walking the oxidative stress tightrope: a perspective from the naked mole-rat, the longest-living rodent. Curr. Pharm. Des., 17, 2290-2307

43. Zhao, S., Lin, L., Kan, G., Xu, C., Tang, Q., Yu, C., Sun, W., Cai, L., Xu, C. and Cui, S. (2014) High autophagy in the naked mole rat may play a significant role in maintaining good health. Cell. Physiol. Biochem., 33, 321-332

44. Buffenstein, R. and Yahav, S. (1991) The effect of diet on microfaunal population and function in the caecum of a subterranean naked mole-rat, Heterocephalus glaber. Br. J. Nutr., $65,249-258$

45. Kim, E. B., Fang, X., Fushan, A. A., Huang, Z., Lobanov, A. V., Han, L., Marino, S. M., Sun, X., Turanov, A. A., Yang, P., et al. (2011) Genome sequencing reveals insights into physiology and longevity of the naked mole rat. Nature, 479, 223-227

46. Fang, X., Nevo, E., Han, L., Levanon, E. Y., Zhao, J., Avivi, A., Larkin, D., Jiang, X., Feranchuk, S., Zhu, Y., et al. (2014) Genome-wide adaptive complexes to underground stresses in blind mole rats Spalax. Nat. Commun., 5, 3966

47. Heintz, C., Doktor, T. K., Lanjuin, A., Escoubas, C. C., Zhang, Y., Weir, H. J., Dutta, S., Silva-García, C. G., Bruun, G. H., Morantte, I., et al. (2017) Splicing factor 1 modulates dietary restriction and TORC1 pathway longevity in C. elegans. Nature, 541, 102-106

48. Wang, E. (2007) MicroRNA, the putative molecular control for mid-life decline. Ageing Res. Rev., 6, 1-11

49. Bell, R., Hubbard, A., Chettier, R., Chen, D., Miller, J. P., Kapahi, P., Tarnopolsky, M., Sahasrabuhde, S., Melov, S. and Hughes, R. E. (2009) A human protein interaction network shows conservation of aging processes between human and invertebrate species. PLoS Genet., 5, e1000414

50. Fernandes, M., Wan, C., Tacutu, R., Barardo, D., Rajput, A., Wang, J., Thoppil, H., Thornton, D., Yang, C., Freitas, A., et al. (2016) Systematic analysis of the gerontome reveals links between aging and age-related diseases. Hum. Mol. Genet., 25, 4804-4818

51. Xue, H., Xian, B., Dong, D., Xia, K., Zhu, S., Zhang, Z., Hou, L., Zhang, Q., Zhang, Y. and Han, J. D. (2007) A modular network model of aging. Mol. Syst. Biol., 3, 147 